\title{
Fast Point Multiplication Algorithms for Binary Elliptic Curves with and without Precomputation
}

\author{
Thomaz Oliveira ${ }^{1(凶)}$, Diego F. Aranha ${ }^{2}$, Julio López ${ }^{2}$, \\ and Francisco Rodríguez-Henríquez ${ }^{1}$ \\ 1 Computer Science Department, CINVESTAV-IPN, Mexico City, Mexico \\ thomaz.figueiredo@gmail.com \\ 2 Institute of Computing, University of Campinas, Campinas, Brazil
}

\begin{abstract}
In this paper we introduce new methods for computing constant-time variable-base point multiplications over the Galbraith-LinScott (GLS) and the Koblitz families of elliptic curves. Using a left-to-right double-and-add and a right-to-left halve-and-add Montgomery ladder over a GLS curve, we present some of the fastest timings yet reported in the literature for point multiplication. In addition, we combine these two procedures to compute a multi-core protected scalar multiplication. Furthermore, we designed a novel regular $\tau$-adic scalar expansion for Koblitz curves. As a result, using the regular recoding approach, we set the speed record for a single-core constant-time point multiplication on standardized binary elliptic curves at the 128-bit security level.
\end{abstract}

Keywords: Binary elliptic curves · Scalar multiplication · Software implementation

\section{Introduction}

From a cryptographic perspective, one of the most interesting consequences of the Snowden revelations is the increased awareness about the importance of implementing security protocols that offer the Perfect Forward Secrecy (PFS) property. The PFS property guarantees that in a given protocol, none of its past short term session keys can be derived from the long term server's private key. One tangible example of this situation is the recent announcement by the Internet Engineering Task Force that the Transport Layer Security (TLS) protocol version 1.3, will no longer include cipher suites based on RSA key transport primitives [34]. Instead, the client-server secret key establishment will be performed via either the Ephemeral Diffie-Hellman or the Elliptic Curve Ephemeral DiffieHellman (ECDHE) methods. Because of the significant performance advantage of the latter over the former, it is anticipated that in the years to come, ECDHE will be the favorite choice for establishing a TLS shared secret.

J. López - The author was supported in part by the Intel Labs University Research Office.

(C) Springer International Publishing Switzerland 2014

A. Joux and A. Youssef (Eds.): SAC 2014, LNCS 8781, pp. 324-344, 2014.

DOI: $10.1007 / 978-3-319-13051-4 \_20$ 
The specifications of all the TLS protocol versions [8-10] include support for prime and binary field elliptic curve cryptographic primitives. In the case of binary elliptic curves, the TLS protocol supports a selection of several standardized random curves as well as Koblitz curves [23] at the 80-, 128-, 192- and 256-bit security levels. Koblitz curves allow performance improvements, due to the availability of the Frobenius automorphism $\tau$. Also, their generation is inherently rigid (in the SafeCurves sense [2]), where the only degree of freedom in the curve generation process consists in choosing a suitable prime degree extension $m$ that produces a curve with almost-prime order. This severely limits the possibility of "1-in-a-million attacks" [35] aiming to reach a weak curve after testing many random seeds.

Point multiplication is the single most important operation of (hyper) elliptic curve cryptography, for that reason, considerable effort has been directed towards achieving fast and compact software/hardware implementations of it. A major result that has influenced the latest implementations was found in 2009, when Galbraith, Lin and Scott (GLS), building on a previous technique introduced by Gallant, Lambert and Vanstone (GLV) [14], constructed efficient endomorphisms for a class of elliptic curves defined over the quadratic field $\mathbb{F}_{q^{2}}$, where $q$ is a prime number [13]. Taking advantage of this result, the authors of [13] performed a 128-bit security level point multiplication that took 326,000 clock cycles on a 64-bit processor. Since then, a steady stream of algorithmic and technological advances has translated into a significant reduction in the number of clock cycles required to compute a (hyper) elliptic curve constant-time variable-base-point multiplication at the 128-bit security level $[1,4,5,11,16,24,38]$.

The authors of $[11,24]$ targeted a twisted Edwards GLV-GLS curve defined over $\mathbb{F}_{p^{2}}$, with $p=2^{127}-5997$. That curve is equipped with a degree-4 endomorphism allowing a fast point multiplication computation that required just 92,000 clock cycles on an Ivy Bridge processor [11]. Bos et al. [5] and Bernstein et al. [1], presented an efficient point multiplication on the Kummer surface associated with the Jacobian of a genus 2 curve defined over a field generated by the prime $p=2^{127}-1$. Each iteration of the Montgomery ladder presented in [1] costs roughly 25 field multiplications, which implemented on a Haswell processor permits to compute a point multiplication in 72,000 clock cycles.

In 2014, Oliveira et al. introduced the $\lambda$-projective coordinate system that leads to faster binary field elliptic curve arithmetic $[31,32]$. The authors applied that coordinate system into a binary GLS curve that admits a degree-2 endomorphism and a fast field arithmetic associated with the quadratic field extension of the binary field $\mathbb{F}_{2^{127}}$. When implemented on a Haswell processor, this approach permits to perform one constant-time point multiplication computation in just 60,000 clock cycles.

Contributions of This Paper. This work presents new methods aimed to perform fast constant-time variable-base-point multiplication computation for both random and Koblitz binary elliptic curves of the form $y^{2}+x y=x^{3}+a x^{2}+b$. In the case of random binary elliptic curves, we introduce a novel right-to-left 
variant of the classical Montgomery-López-Dahab ladder algorithm presented in [25], which efficiently adapted the original ladder idea introduced by Peter Montgomery in his 1987 landmark paper [26]. The new variant presented in this work does not require point doublings, but instead, it uses the efficient point halving operation available on binary elliptic curves. In contrast with the algorithm presented in [25] that does not admit the benefit of precomputed tables, our proposed variant can take advantage of this technique, a feature that could be proved valuable for the fixed-base-point multiplication scenario. Moreover, we show that our new right-to-left Montgomery ladder formulation can be nicely combined with the classical ladder to attain a high parallel acceleration factor for a constant-time multi-core implementation of the point multiplication operation. As a second contribution, we present a procedure that adapts the regular scalar recoding of [21] to the task of producing a regular $\tau$-NAF scalar recoding for Koblitz curves. This approach has faster precomputation than related recodings [30] and allows us to achieve a speed record for single-core constant-time point multiplication on standardized binary elliptic curves at the 128-bit security level.

The remainder of this paper is organized as follows. In Sect. 2 we give a short description of the GLS and Koblitz curves, their arithmetic and their security. In Sect. 3 we present new variants of the Montgomery ladder for binary elliptic curves. Then, in Sect. 4 , we introduce a regular $\tau$-NAF recoding amenable for producing protected point multiplication implementations on Koblitz curves. In Sect. 5, we present our experimental implementation results and finally, we draw our conclusions in Sect. 6.

\section{Mathematical Background}

\subsection{Quadratic Field Arithmetic}

A binary extension field $\mathbb{F}_{q}, q=2^{m}$, can be constructed by taking an degree- $m$ polynomial $f(x) \in \mathbb{F}_{2}[x]$ irreducible over $\mathbb{F}_{2}$, where the field elements in $\mathbb{F}_{q}$ are the set of binary polynomials of degree less than $m$. Quadratic extensions of a binary extension field can be built using a degree two monic polynomial $g(u) \in$ $\mathbb{F}_{q}[u]$ irreducible over $\mathbb{F}_{q}$. In this case, the field $\mathbb{F}_{q^{2}}$ is isomorphic to $\mathbb{F}_{q}[u] /(g(u))$ and its elements can be represented as $a_{0}+a_{1} u$, with $a_{0}, a_{1} \in \mathbb{F}_{q}$. Operations in the quadratic extension are performed coefficient-wise. For instance, the multiplication of two elements $a, b \in \mathbb{F}_{q^{2}}$ is computed at the cost of three multiplications in the base field using the customary Karatsuba formulation,

$$
\begin{aligned}
a \cdot b & =\left(a_{0}+a_{1} u\right) \cdot\left(b_{0}+b_{1} u\right) \\
& =\left(a_{0} b_{0}+a_{1} b_{1}\right)+\left(a_{0} b_{0}+\left(a_{0}+a_{1}\right) \cdot\left(b_{0}+b_{1}\right)\right) u
\end{aligned}
$$

with $a_{0}, a_{1}, b_{0}, b_{1} \in \mathbb{F}_{q}$.

In $[31,32]$, the authors developed an efficient software library for the field $\mathbb{F}_{2^{m}}$ and its quadratic extension $\mathbb{F}_{2^{2 m}}$, with $m=127$, generated by means of the irreducible trinomials $f(x)=x^{127}+x^{63}+1$ and $g(u)=u^{2}+u+1$, respectively. 
The computational cost of the field arithmetic in the quadratic extension field gets significantly reduced by using that towering approach. To be more concrete, let $M$ and $m$ denote the cost of one field multiplication over $\mathbb{F}_{q^{2}}$ and $\mathbb{F}_{q}$, respectively. The execution of the arithmetic library of [32] on the Sandy Bridge and Haswell microprocessors yields a ratio $M / m$ of just 2.23 and 1.51 , respectively. These experimental results are considerably better than the theoretical ratio $M / m=3$ that one could expect from the Karatsuba formulation of Eq. (1). The aforementioned performance speedup can be explained from the fact that the towering field approach permits a much better usage of the processor's pipelined execution unit, which potentially can improve the speed of one 64-bit carry-less multiplication $^{1}$ from 7 clock cycles to the maximum achievable throughput of just 2 clock cycles [12].

\subsection{GLS Binary Elliptic Curves}

Let $E_{a, b}\left(\mathbb{F}_{q^{2}}\right)$ denote the additive abelian group formed by the point at infinity $\mathcal{O}$ and the set of affine points $P=(x, y)$ with $x, y \in \mathbb{F}_{q^{2}}$ that satisfy the ordinary binary elliptic curve equation given as,

$$
E: y^{2}+x y=x^{3}+a x^{2}+b,
$$

defined over $\mathbb{F}_{q^{2}=2^{2 m}}$, with $a \in \mathbb{F}_{q^{2}}$ and $b \in \mathbb{F}_{q^{2}}^{*}$. Let $\# E_{a, b}\left(\mathbb{F}_{q^{2}}\right)$ denote the size of the group $E_{a, b}\left(\mathbb{F}_{q^{2}}\right)$, and let us assume that $E_{a, b}\left(\mathbb{F}_{q^{2}}\right)$ includes a subgroup $\langle P\rangle$ of prime order $r$.

The point multiplication operation, denoted by $Q=k P$, corresponds to adding $P$ to itself $k-1$ times, with $k \in[0, r-1]$. The average cost of computing $k P$ by a random $n$-bit scalar $k$ using the traditional double-and-add method is about $n D+\frac{n}{2} A$, where $D$ and $A$ are the cost of doubling and adding a point, respectively. If the elliptic curve $E$ of Eq. (2) is equipped with a non-trivial efficiently computable endomorphism $\psi$ such that $\psi(P)=\delta P \in\langle P\rangle$, for some $\delta \in[2, r-2]$. Then the point multiplication can be computed $\grave{a}$ la GLV as,

$$
Q=k P=k_{1} P+k_{2} \psi(P)=k_{1} P+k_{2} \cdot \delta P,
$$

where the subscalars $\left|k_{1}\right|,\left|k_{2}\right| \approx n / 2$, can be found by solving a closest vector problem in a lattice [13]. Having split the scalar $k$ into two parts, the computation of $k P=k_{1} P+k_{2} \psi(P)$ can be performed by applying simultaneous multiple point multiplication techniques [18] that translates into a saving of half of the doublings required by the execution of a single point multiplication $k P$.

Inspired by the GLS technique of [13], Hankerson, Karabina and Menezes presented in [17] a family of binary GLS curves defined over the field $\mathbb{F}_{q^{2}}$, with $q=2^{m}$, which admits a two-dimensional endomorphism. This endomorphism can be computed at the inexpensive cost of just three additions in $\mathbb{F}_{q}$. Furthermore, by carefully choosing the elliptic curve parameters $a, b$ of Eq. (2), the authors of [17] showed that it is possible to find members of that family of GLS curves with an almost-prime group order of the form $\# E_{a, b}\left(\mathbb{F}_{q^{2}}\right)=h r$, with $h=2$ and where $r$ is a $(2 m-1)$-bit prime number.

${ }^{1}$ Corresponding to the Intel's PCLMULQDQ instruction. 
Security of GLS Curves. Given a point $Q \in\langle P\rangle$, the Elliptic Curve Discrete Logarithm Problem (ECDLP) consists of finding the unique integer $k \in[0, r-1]$ such that $Q=k P$. To the best of our knowledge, the most powerful attack for solving the ECDLP on binary elliptic curves was presented in [33] (see also $[20,36])$, with an associated computational complexity of $O\left(2^{c \cdot m^{2 / 3}} \log m\right)$, where $c<2$, and where $m$ is a prime number. This is worse than generic algorithms with time complexity $O\left(2^{m / 2}\right)$ for all prime field extensions $m$ less than $N=2000$, a bound that is well above the range used for performing elliptic curve cryptography [33]. On the other hand, since the elliptic curve of Eq. (2) is defined over a quadratic extension of the field $\mathbb{F}_{q}$, the generalized Gaudry-HessSmart (gGHS) attack $[15,19]$ to solve the ECDLP on the curve $E$, applies. To prevent this attack, it suffices to verify that the constant $b$ of $E_{a, b}\left(\mathbb{F}_{q^{2}}\right)$ is not weak. Nevertheless, the probability that a randomly selected $b \in \mathbb{F}_{q}^{*}$ is a weak parameter, is negligibly small [17].

\subsection{Koblitz Curves}

A Koblitz curve, also known as an anomalous binary curve or subfield curve, is defined as the set of affine points $P=(x, y) \in \mathbb{F}_{q} \times \mathbb{F}_{q}, q=2^{m}$, that satisfy the Weierstraß equation $E_{a}: y^{2}+x y=x^{3}+a x^{2}+1, a \in\{0,1\}$, together with a point at infinity denoted by $\mathcal{O}$. In $\lambda$-affine coordinates, where the points are represented as $P=\left(x, \lambda=x+\frac{y}{x}\right), x \neq 0$, the $\lambda$-affine form of the above equation becomes [32], $\left(\lambda^{2}+\lambda+a\right) x^{2}=x^{4}+1$. A Koblitz curve forms an abelian group denoted as $E_{a}\left(\mathbb{F}_{2^{m}}\right)$ of order $2(2-a) r$, for an odd prime $r$, where its group law is defined by the point addition operation.

Frobenius Map. Since their introduction in [23], Koblitz curves were extensively studied for their additional structure that allows, in principle, a performance speedup in the point multiplication computation. The Frobenius map $\tau: E_{a}\left(\mathbb{F}_{q}\right) \rightarrow E_{a}\left(\mathbb{F}_{q}\right)$ defined by $\tau(\mathcal{O})=\mathcal{O}, \tau(x, y)=\left(x^{2}, y^{2}\right)$, is a curve automorphism satisfying $\left(\tau^{2}+2\right) P=\mu \tau(P)$ for $\mu=(-1)^{1-a}$ and all $P \in E_{a}\left(\mathbb{F}_{q}\right)$. By solving the equation $\tau^{2}+2=\mu \tau$, the Frobenius map can be seen as the complex number $\tau=\frac{\mu+\sqrt{-7}}{2}$. Notice that in $\lambda$-coordinates the Frobenius map action remains the same, because, $\tau(x, \lambda)=\left(x^{2}, \lambda^{2}\right)=\left(x^{2}, x^{2}+\frac{y^{2}}{x^{2}}\right)$, which corresponds to the $\lambda$-representation of $\tau(x, y)$. Let $\mathbb{Z}[\tau]$ be the ring of polynomials in $\tau$ with coefficients in $\mathbb{Z}$. Since the Frobenius map is highly efficient, as long as it is possible to convert an integer scalar $k$ to its $\tau$-representation $k=\sum_{i=0}^{l-1} u_{i} \tau^{i}$, its action can be exploited in a point multiplication computation by adding multiples $u_{i} \tau^{i}(P)$, with $u_{i} \tau^{i} \in \mathbb{Z}[\tau]$. Solinas [37] proposed exactly that, namely, a $\tau$-adic scalar recoding analogous to the signed digit scalar Non-Adjacent Form representation.

Security of Koblitz Curves. From the security point of view, it has been argued that the availability of additional structure in the form of endomorphisms can be a potential threat to the hardness of elliptic curve discrete logarithms [3], 
but limitations observed in approaches based on isogeny walks is evidence contrariwise [22]. Furthermore, the generation of Koblitz curves satisfy by definition the rigidity property. Constant-time compact implementations for Koblitz curves are also easily obtained by specializing the Montgomery-López-Dahab ladder algorithm [25] for $b=1$, although we show below that this is not the most efficient constant-time implementation strategy possible. Another practical advantage is the adoption of Koblitz curves by several standards bodies [27], which guarantee interoperability and availability of implementations in many hardware and software platforms.

\section{New Montgomery Ladder Variants}

This Section presents algorithms for computing the scalar multiplication through the Montgomery ladder method. Here, we let $P$ be a point in a binary elliptic curve of prime order $r>2$ and $k$ a scalar of bit length $n$. Our objective is to compute $Q=k P$.

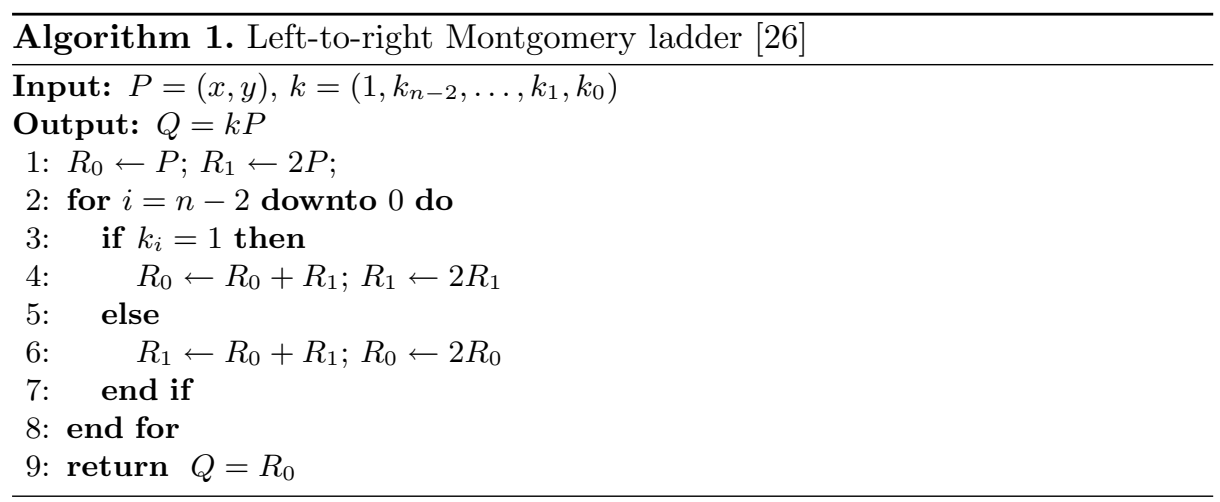

Algorithm 1 describes the classical left-to-right Montgomery ladder approach for point multiplication [26], whose key algorithmic idea is based on the following observation. Given a base point $P$ and two input points $R_{0}$ and $R_{1}$, such that their difference, $R_{0}-R_{1}=P$, is known, the $x$-coordinates of the points, $2 R_{0}$, $2 R_{1}$ and $R_{0}+R_{1}$, are fully determined by the $x$-coordinates of $P, R_{0}$ and $R_{1}$.

More than one decade after its original proposal in [26], López and Dahab presented in [25] an optimized version of the Montgomery ladder, which was specifically crafted for the efficient computation of point multiplication on ordinary binary elliptic curves. In this scenario, compact formulae for the point addition and point doubling operations of Algorithm 1 can be derived from the following result. 
Lemma 1 [25]. Let $P=(x, y), R_{1}=\left(x_{1}, y_{1}\right)$, and $R_{0}=\left(x_{0}, y_{0}\right)$ be elliptic curve points, and assume that $R_{1}-R_{0}=P$, and $x_{0} \neq 0$. Then, the $x$-coordinate of the point $\left(R_{0}+R_{1}\right), x_{3}$, can be computed in terms of $x_{0}, x_{1}$, and $x$ as follows,

$$
x_{3}= \begin{cases}x+\frac{x_{0} \cdot x_{1}}{\left(x_{0}+x_{1}\right)^{2}} & R_{0} \neq \pm R_{1} \\ x_{0}^{2}+\frac{b}{x_{0}^{2}} & R_{0}=R_{1}\end{cases}
$$

Moreover, the $y$-coordinate of $R_{0}$ can be expressed in terms of $P$, and the $x$ coordinates of $R_{0}, R_{1}$ as,

$$
y_{0}=x^{-1}\left(x_{0}+x\right)\left[\left(x_{0}+x\right)\left(x_{1}+x\right)+x^{2}+y\right]+y
$$

Let us denote the projective representation of the points $R_{0}, R_{1}$ and $R_{0}+R_{1}$, without considering their $y$-coordinates as, $R_{0}=\left(X_{0},-, Z_{0}\right), R_{1}=\left(X_{1},-, Z_{1}\right)$ and $R_{0}+R_{1}=\left(X_{3},-, Z_{3}\right)$. Then, for the case $R_{0}=R_{1}$, Lemma 1 implies,

$$
\left\{\begin{array}{l}
X_{3}=X_{0}^{4}+b \cdot Z_{0}^{4} \\
Z_{3}=X_{0}^{2} \cdot Z_{0}^{2}
\end{array}\right.
$$

Furthermore, for the case $R_{0} \neq \pm R_{1}$, one has that,

$$
\left\{\begin{array}{l}
Z_{3}=\left(X_{0} \cdot Z_{1}+X_{1} \cdot Z_{0}\right)^{2} \\
X_{3}=x \cdot Z_{3}+\left(X_{0} \cdot Z_{1}\right) \cdot\left(X_{1} \cdot Z_{0}\right)
\end{array}\right.
$$

From Eqs. (5) and (6) it follows that the computational cost of each ladder step in Algorithm 1 is of 5 multiplications, 1 multiplication by the curve $b$-constant, 4 or 5 squarings $^{2}$ and 3 additions over the binary extension field where the elliptic curve has been defined.

In the rest of this Section, we will present a novel right-to-left formulation of the classical Montgomery ladder.

\subsection{Right-to-Left Double-and-Add Montgomery-LD Ladder}

Algorithm 2 presents a right-to-left version of the classical Montgomery ladder procedure. At the end of the $i$-th iteration, the points in the variables $R_{0}, R_{1}$ are, $R_{0}=2^{i+1} P$, and $R_{1}=\ell P+\frac{P}{2}$, where $\ell$ is the integer represented by the $i$ rightmost bits of the scalar $k$. The variable $R_{2}$ maintains the relationship, $R_{2}=R_{0}-R_{1}$ from the initialization (step 1), until the execution of the last iteration of the main loop (steps 2-9). This comes from the fact that at each iteration, if $k_{i}=1$, then the difference $R_{0}-R_{1}$ remains unchanged. If otherwise, $k_{i}=0$, then both $R_{2}$ and $R_{0}$ are updated with their respective original values plus $R_{0}$, which ensures that $R_{2}=R_{0}-R_{1}$, still holds. Notice however that, although the difference $R_{2}=R_{0}-R_{1}$, is known, it may vary throughout the iterations.

${ }^{2}$ Either $b=1$ or $\sqrt{b}$ is precomputed. Formula (5) can also be computed as $Z_{3}=$ $\left(X_{0} \cdot Z_{0}\right)^{2}$ and $X_{3}=\left(X_{0}^{2}+\sqrt{b} \cdot Z_{0}^{2}\right)^{2}$. 


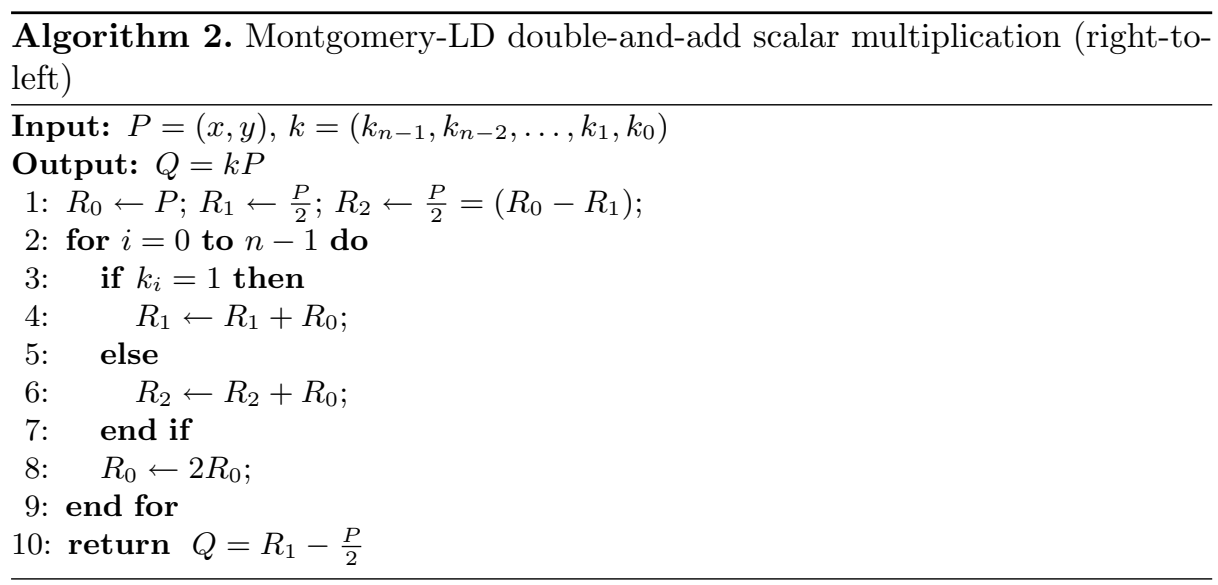

As stated in Lemma 1, the point additions of steps 4 and 6 in Algorithm 2 can be computed using the $x$-coordinates of the points $R_{0}, R_{1}$ and $R_{2}$, according to the following analysis. If $k_{i}=1$, then the $x$-coordinate of $R_{0}+R_{1}$ is a function of the $x$-coordinates of $R_{0}, R_{1}$ and $R_{2}$, because $R_{2}=R_{0}-R_{1}$. If $k_{i}=0$, the $x$-coordinate of $R_{2}+R_{0}$ is a function of the $x$-coordinates of the points $R_{0}$, $R_{1}$ and $R_{2}$, because $R_{0}-R_{2}=R_{0}-\left(R_{0}-R_{1}\right)=R_{1}$. Hence, considering the projective representation of the points $R_{0}=\left(X_{0},-, Z_{0}\right), R_{1}=\left(X_{1},-, Z_{1}\right)$, $R_{2}=\left(X_{2},-, Z_{2}\right)$ and $R_{0}+R_{1}=\left(X_{3},-, Z_{3}\right)$, where all the $y$-coordinates are ignored, and assuming $R_{0} \neq \pm R_{1}$, we have,

$$
\left\{\begin{array}{l}
T=\left(X_{0} \cdot Z_{1}+X_{1} \cdot Z_{0}\right)^{2} \\
Z_{3}=Z_{2} \cdot T \\
X_{3}=X_{2} \cdot T+Z_{2} \cdot\left(X_{0} \cdot Z_{1}\right) \cdot\left(X_{1} \cdot Z_{0}\right)
\end{array}\right.
$$

From Eqs. (5) and (7), it follows that the computational cost of each ladder step in Algorithm 2 is of 7 multiplications, 1 multiplication by the curve $b$-constant, 4 or 5 squarings and 3 additions over the binary field where the elliptic curve lies.

Although conceptually simple, the above method has several algorithmic and practical shortcomings. The most important one is the difficulty to recover, at the end of the algorithm, the $y$-coordinate of $R_{1}$, as in none of the available points $\left(R_{0}, R_{1}\right.$ and $R_{2}$ ) the corresponding $y$-coordinate is known. This may force the decision to use complete projective formulae for the point addition and doubling operations of steps 4,6 and 8, which would be costly. Finally, we stress that to guarantee that the case $R_{0}=R_{2}$ will never occur, it is sufficient to initialize $R_{1}$ with $\frac{P}{2}$, and perform an affine subtraction at the end of the main loop (step 10).

In the following subsection we present a halve-and-add right-to-left Montgomery ladder algorithm that alleviates the above shortcomings and still achieves a competitive performance. 


\subsection{Right-to-Left Halve-and-Add Montgomery-LD Ladder}

Algorithm 3 presents a right-to-left Montgomery ladder procedure similar to Algorithm 2, but in this case, all the point doubling operations are substituted with point halvings. A left-to-right approach using halve-and-add with Montgomery ladder was published in [29], however, this method requires one inversion per iteration, which degrades its efficiency due to the cost of this operation.

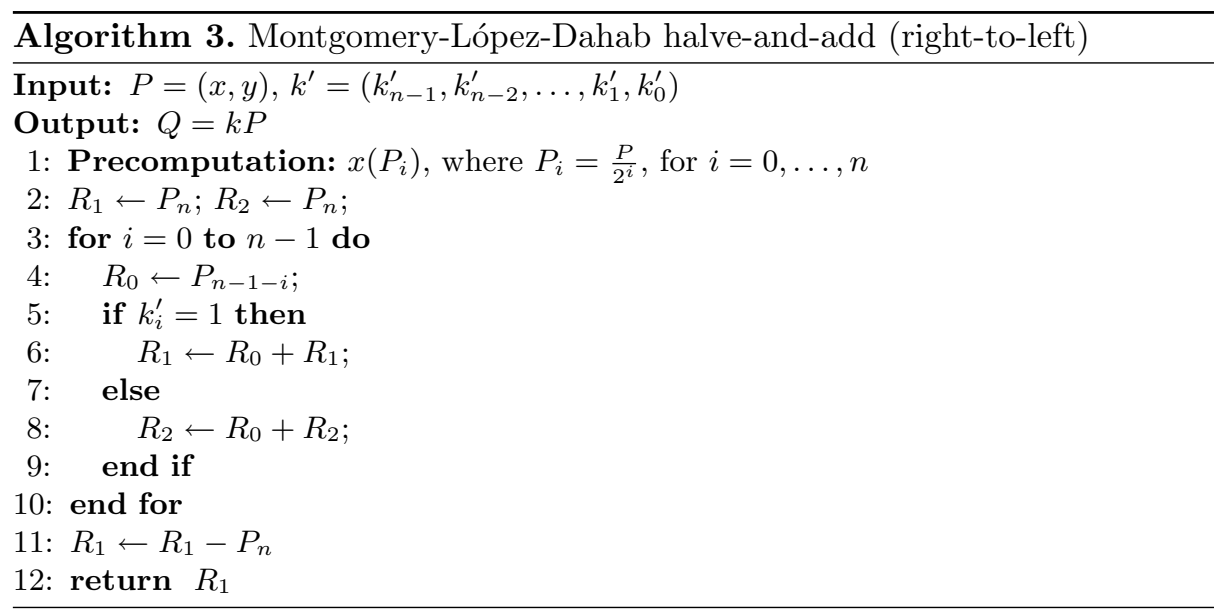

As in any halve-and-add procedure, an initial step before performing the actual computation consists of processing the scalar $k$ such that it can be equivalently represented with negative powers of two. To this end, one first computes $k^{\prime} \equiv 2^{n-1} k \bmod r$, with $n=|r|$. This implies that, $k \equiv \sum_{i=1}^{n} k_{n-i}^{\prime} / 2^{i-1} \bmod r$ and therefore, $k P=\sum_{i=1}^{n} k_{n-i}^{\prime}\left(\frac{1}{2^{i-1}} P\right)$. Then, in the first step of Algorithm 3, $n$ halvings of the base point $P$ are computed. We stress that all the precomputed points $P_{i}=\frac{P}{2^{i}}$, for $i=0, \ldots, n$ can be stored in affine coordinates. In fact, just the $x$-coordinate of each one of the above $n$ points must be stored (with the sole exception of the point $P_{n}$, whose $y$-coordinate is also computed and stored).

As in the preceding algorithm notice that at the end of the $i$-th iteration, the points in the variables $R_{0}, R_{1}$ are, $R_{0}=\frac{P}{2^{n-i-1}}$, and $R_{1}=\ell P+P_{n}$, where in this case $\ell$ is the integer represented as, $\ell=\sum_{j=0}^{i} \frac{k_{j}^{\prime}}{2^{n-j}} \bmod r$. Notice also that the variable $R_{2}$ maintains the relationship, $R_{2}=R_{0}-R_{1}$, until the execution of the last iteration of the main loop (steps $3-10$ ). This comes from the fact that at each iteration, if $k_{i}=1$, then the difference $R_{0}-R_{1}$ remains unchanged. If otherwise, $k_{i}=0$, then both $R_{2}$ and $R_{0}$ are updated with their respective original values plus $R_{0}$, which ensures that $R_{2}=R_{0}-R_{1}$, still holds.

Since at every iteration, the values of the points $R_{0}, R_{1}$ and $R_{0}-R_{1}$, are all known, the compact point addition formula (7) can be used. In practice, this is also possible because the $y$-coordinate of the output point $k P$ can be readily 
recovered using Eq. 4, along with the point $2 P$. Moreover, since the points in the precomputed table were generated using affine coordinates, it turns out that the $z$-coordinate of the point $R_{0}$ is always 1 for all the iterations of the main loop. This simplifies (7) as,

$$
\left\{\begin{array}{l}
T=\left(X_{0} \cdot Z_{1}+X_{1}\right)^{2} \\
Z_{3}=Z_{2} \cdot T \\
X_{3}=X_{2} \cdot T+Z_{2} \cdot\left(X_{0} \cdot Z_{1}\right) \cdot\left(X_{1}\right)
\end{array}\right.
$$

Hence, the computational cost per iteration of Algorithm 3 is of 5 multiplications, 1 squaring, 2 additions and one point halving over the binary field where the elliptic curve lies.

GLS Endomorphism. The efficient computable endomorphism provided by the GLS curves can be used to implement the 2-GLV method on the Algorithm 3. As a result, only $n / 2$ point halving operations must be computed. Besides the speed improvement, the $2-$ GLV method reduces to a half the number of precomputed points that must be stored.

\subsection{Multi-core Montgomery Ladder}

As proposed in [38], by properly recoding the scalar, one can efficiently compute the scalar multiplication in a multi-core environment. Specifically, given a scalar $k$ of size $n$, we fix a constant $t$ which establishes how many scalar bits will be processed by the double-and-add, and by the halve-and-add procedures. This is accomplished by computing, $k^{\prime}=2^{t} k \bmod r$, which yields,

$$
k=\underbrace{\frac{k_{0}^{\prime}}{2^{t}}+\frac{k_{1}^{\prime}}{2^{t-1}}+\cdots+\frac{k_{t-1}^{\prime}}{2^{1}}}_{\text {halve-and-add }}+\underbrace{\frac{k_{t}^{\prime}}{2^{0}}+2^{1} k_{t+1}^{\prime}+2^{2} k_{t+2}^{\prime}+\cdots+2^{(n-1)-t} k_{n-1}^{\prime}}_{\text {double-and-add }}
$$

In a two-core setting, it is straightforward to combine the left-to-right and right-to-left Montgomery ladder procedures of Algorithms 1 and 3, and distribute them to both cores. In this scenario, the number of necessary pre-computed halved points reduces to $\sim \frac{n}{4}$. In a four-core platform, we can apply the GLS endomorphism to the left-to-right Montgomery ladder (Algorithm 1). Even though the GLV technique is ineffective for the classical Montgomery algorithm (due to the fact that we cannot share the point doublings between the base point and its endomorphism), the method permits an efficient splitting of the algorithm workload into two cores. In this way, one can use the first two cores for computing $t$-digits of the GLV subscalars $k_{1}$ and $k_{2}$ by means of Algorithm 3 , while we allocate the other two cores to compute the rest of the scalar's bits using Algorithm 1, as shown in Algorithm 6 (see Appendix A). 
Table 1. Montgomery-LD algorithms cost comparison. In this table, $M, M_{a}, M_{b}, S, I$ denote the following field operations: multiplication, multiplication by the curve $a$ constant, multiplication by the curve $b$-constant, squaring and inversion. The point halving operation is denoted by $H$.

\begin{tabular}{|c|c|c|c|c|}
\hline & Method & & & Cost \\
\hline $\begin{array}{l}0 \\
\vdots \\
\vdots \\
\vdots \\
-1 \\
\end{array}$ & \begin{tabular}{|l|}
$\begin{array}{l}\text { Alg. 1: Montgomery-LD } \\
\text { (double-and-add, left-to-right) }\end{array}$ \\
$\begin{array}{l}\text { Alg. 3: Montgomery-LD-2-GLV } \\
\text { (halve-and-add, right-to-left) }\end{array}$ \\
\end{tabular} & & $\begin{array}{l}\text { pre/post } \\
\text { sc. mult. } \\
\text { pre/post } \\
\text { sc. mult. }\end{array}$ & $\begin{array}{c}10 M+1 S+1 I \\
n\left(5 M+1 M_{b}+4 S\right) \\
48 M+1 M_{a}+13 S+3 I \\
\left(\frac{n}{2}+1\right) H+n(5 M+1 S) \\
\end{array}$ \\
\hline & $\begin{array}{l}\text { Montgomery-LD-2-GLV } \\
\text { (double-and-add, left-to-right) } \\
\text { Montgomery-LD-2-GLV } \\
\text { (halve-and-add, right-to-left) } \\
\text { Overhead }\end{array}$ & $\begin{array}{l}\text { core I } \\
\text { core II }\end{array}$ & $\begin{array}{l}\text { pre/post } \\
\text { sc. mult. } \\
\text { pre/post } \\
\text { sc. mult. }\end{array}$ & $\begin{array}{c}25 M+1 M_{a}+5 S+2 I \\
\left(n-t_{2}\right)\left(5 M+1 M_{b}+4 S\right) \\
46 M+2 M_{a}+12 S+2 I \\
\left.\frac{t_{2}}{2}+1\right) H+t_{2}(5 M+1 S) \\
15 M+5 S+1 I\end{array}$ \\
\hline $\begin{array}{l}0 \\
: 0 \\
\dot{0} \\
\dot{H}\end{array}$ & $\begin{array}{l}\text { Montgomery-LD-2-GLV } \\
\text { (double-and-add, left-to-right) } \\
\text { Montgomery-LD-2-GLV } \\
\text { (halve-and-add, right-to-left) } \\
\text { Overhead }\end{array}$ & $\begin{array}{l}\text { cores } \\
\text { I \& II } \\
\text { cores } \\
\text { III \& IV }\end{array}$ & $\begin{array}{l}\text { pre/post } \\
\text { sc. mult. } \\
\text { pre/post } \\
\text { sc. mult. }\end{array}$ & $\begin{array}{c}10 M+1 S+1 I \\
\left(\frac{n}{2}-t_{4}\right)\left(5 M+1 M_{b}+4 S\right) \\
16 M+1 M_{a}+4 S+1 I \\
\frac{\left(\frac{t_{4}}{2}+1\right) H+t_{4}(5 M+1 S)}{34 M+1 M_{a}+12 S+1 I} \\
\end{array}$ \\
\hline
\end{tabular}

\subsection{Cost Comparison of Montgomery Ladder Variants}

Table 1 shows the computational costs associated to the Montgomery ladder variants described in this Section. The constants $t_{2}$ and $t_{4}$ represent the values of the parameter $t$ chosen for the two- and four-core implementations, respectively. ${ }^{3}$ All Montgomery ladder algorithms require a basic post-computation cost to retrieve the $y$-coordinate, which demands ten multiplications, one squaring and one inversion. Due to the application of the GLV technique, the Montgomery-LD-2-GLV halve-and-add version (corresponding to Algorithm 3), requires some few extra operations, namely, the subtraction of a point and the addition of two accumulators, which is performed using the López-Dahab (LD) projective coordinate formulae. In the end, one extra inversion is needed to convert the point representation from LD-projective coordinates to affine coordinates.

In the case of the parallel versions, the overhead is given by the post-computation done in one single core. The exact costs are mainly determined by the accumulator additions that are performed via full and mixed LDprojective formulae. In all of the timings reported in Sect.5, we consider the LD-projective to affine coordinate transformation cost.

\section{A Novel Regular $\tau$-Adic Approach}

\subsection{Recoding in $\tau$-Adic Form}

The recoding approach proposed by Solinas finds an element $\rho \in \mathbb{Z}[\tau]$, of as small norm as possible, such that $\rho \equiv k\left(\bmod \frac{\tau^{m}-1}{\tau-1}\right)$. A $\tau$-adic expansion with

\footnotetext{
${ }^{3}$ In our implementations (see Subsect. 5.3 below), the values used for the parameters $t_{2}$ and $t_{4}$ ranged from 53 to 55 .
} 


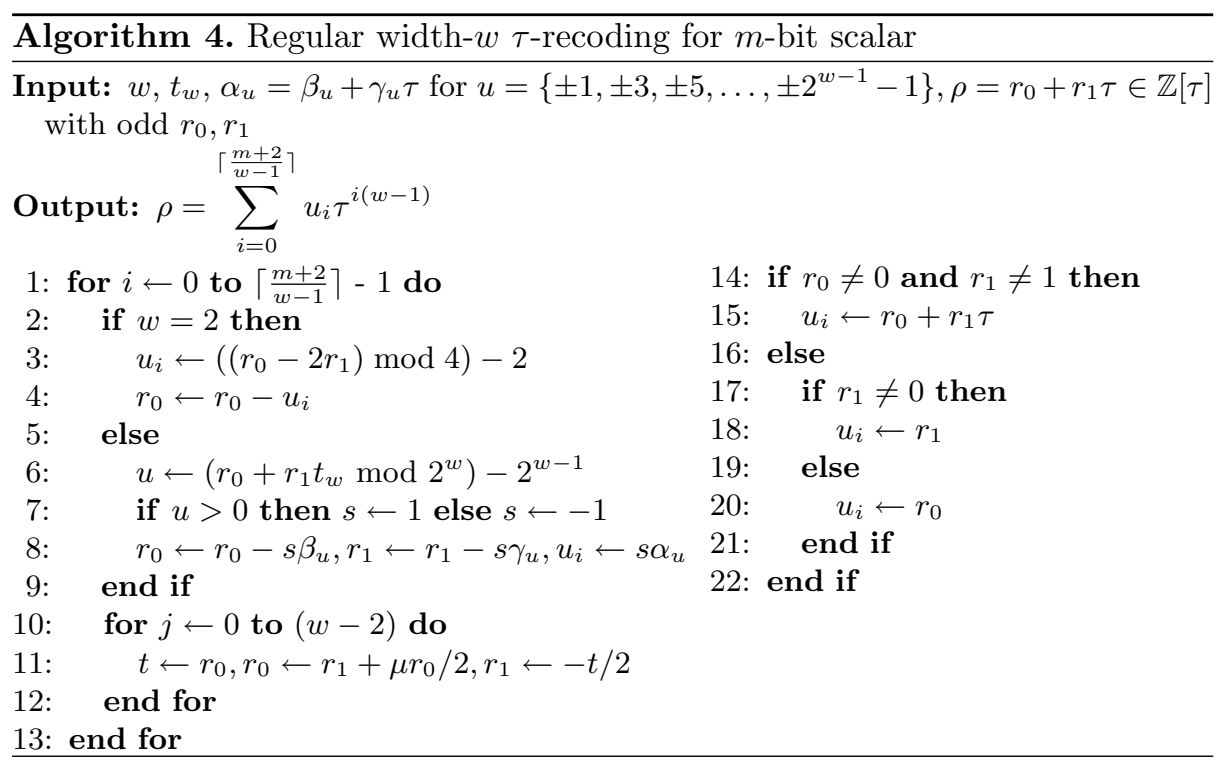

average non-zero density $\frac{1}{3}$ can be obtained by repeatedly dividing $\rho$ by $\tau$ and assigning the remainders to the digits $u_{i}$ to obtain $k=\sum_{i=0}^{i=l-1} u_{i} \tau^{i}$. An alternative approach that does not involve multi-precision divisions, is to compute an element $\rho^{\prime}=k \operatorname{partmod}\left(\frac{\tau^{m}-1}{\tau-1}\right)$ by performing a partial reduction procedure [37]. A width- $w \tau$-NAF expansion with non-zero density $\frac{1}{w+1}$, where at most one of any $w$ consecutive coefficients is non-zero, can also be obtained by repeatedly dividing $\rho^{\prime}$ by $\tau^{w}$ and assigning the remainders to the digit set $\left\{0, \pm \alpha_{1}, \pm \alpha_{3}, \ldots, \pm \alpha_{2^{w-1}-1}\right\}$, for $\alpha_{i}=i \bmod \tau^{w}$. Under reasonable assumptions, this window-based recoding has length $l \leq m+1$ [37].

In this section, a regular recoding version of the (width- $w$ ) $\tau$-NAF expansion is derived. The security advantages of such recoding are the predictable length and locations of non-zero digits in the expansion. This eliminates any side-channel information that an attacker could possibly collect regarding the operation executed at any iteration of the scalar multiplication algorithm (point doubling/Frobenius map or point addition). As long as querying a precomputed table of points to select the second operand of a point addition takes constant time, the resulting algorithm should be resistant against any timing-based sidechannel attacks.

Let us first consider the integer recoding proposed by Joye and Tunstall [21]. They observed that any odd integer $i$ in the interval $\left[0,2^{w}\right)$ can be written as $i=2^{w-1}+\left(-\left(2^{w-1}-i\right)\right)$. Repeatedly dividing an odd $n$-bit integer $k-$ $\left(\left(k \bmod 2^{w}\right)-2^{w-1}\right)$ by $2^{w-1}$ maintains the parity and assigns the remainders to the digit set $\left\{ \pm 1, \ldots, \pm\left(2^{w-1}-1\right)\right\}$, producing an expansion of length $\left\lceil 1+\frac{n}{w-1}\right]$ with non-zero density $\frac{1}{w-1}$. Our solution for the problem of finding a regular $\tau$-adic expansion employs the same intuition, as explained next. 
Let $\phi_{w}: \mathbb{Z}[\tau] \rightarrow \mathbb{Z}_{2^{w}}$ be a surjective ring homomorphism induced by $\tau \mapsto t_{w}$, for $t_{w}^{2}+2 \equiv \mu t_{w}\left(\bmod 2^{w}\right)$, with kernel $\left\{\alpha \in \mathbb{Z}[\tau]: \tau^{w}\right.$ divides $\left.\alpha\right\}$. An element $i=i_{0}+i_{1} \tau$ from $\mathbb{Z}[\tau]$ with odd integers $i_{0}, i_{1} \in\left[0,2^{w}\right)$ satisfies the analogous property $\phi_{w}(i)=2^{w-1}+\left(-\left(2^{w-1}-\phi_{w}(i)\right)\right)$. Repeated division of $\left(r_{0}+r_{1} \tau\right)-$ $\left(\left(\left(r_{0}+r_{1} \tau\right) \bmod \tau^{w}\right)-\tau^{w-1}\right)$ by $\tau^{w-1}$, correspondingly of $\phi_{w}\left(\rho^{\prime}\right)=\left(r_{0}+\right.$ $\left.r_{1} t_{w}\right)-\left(\left(r_{0}+r_{1} t_{w} \bmod 2^{w}\right)-2^{w-1}\right)$ by $2^{w-1}$, obtains remainders that belong to the set $\left\{0, \pm \alpha_{1}, \pm \alpha_{3}, \ldots, \pm \alpha_{2^{w-1}-1}\right\}$. The resulting expansion always has length $\left\lceil 1+\frac{m+2}{w-1}\right\rceil$ and non-zero density $\frac{1}{w-1}$. Algorithm 4 presents the recoding process for any $w \geq 2$. The resulting recoding can also be seen as an adaption of the SPAresistant recoding of [30], mapping to the digit set $\left\{0, \pm \alpha_{1}, \pm \alpha_{3}, \ldots, \pm \alpha_{2^{w-1}-1}\right\}$ instead of integers. While the non-zero densities are very similar, our scheme provides a performance benefit in the precomputation step, since the Frobenius map is usually faster than point doubling and preserves affine coordinates and consequently faster point additions.

\subsection{Left-to-Right Regular Approach}

Algorithm 5 presents a complete description of a regular scalar multiplication approach that uses as a building block the regular width- $w \tau$-recoding procedure just described.

For benchmarking purposes, we also included a baseline implementation of the customary Montgomery López-Dahab ladder. This allows easier comparisons with related work and permits to evaluate the impact of incomplete reduction in the field arithmetic performance (cf. Subsect. 5.2).

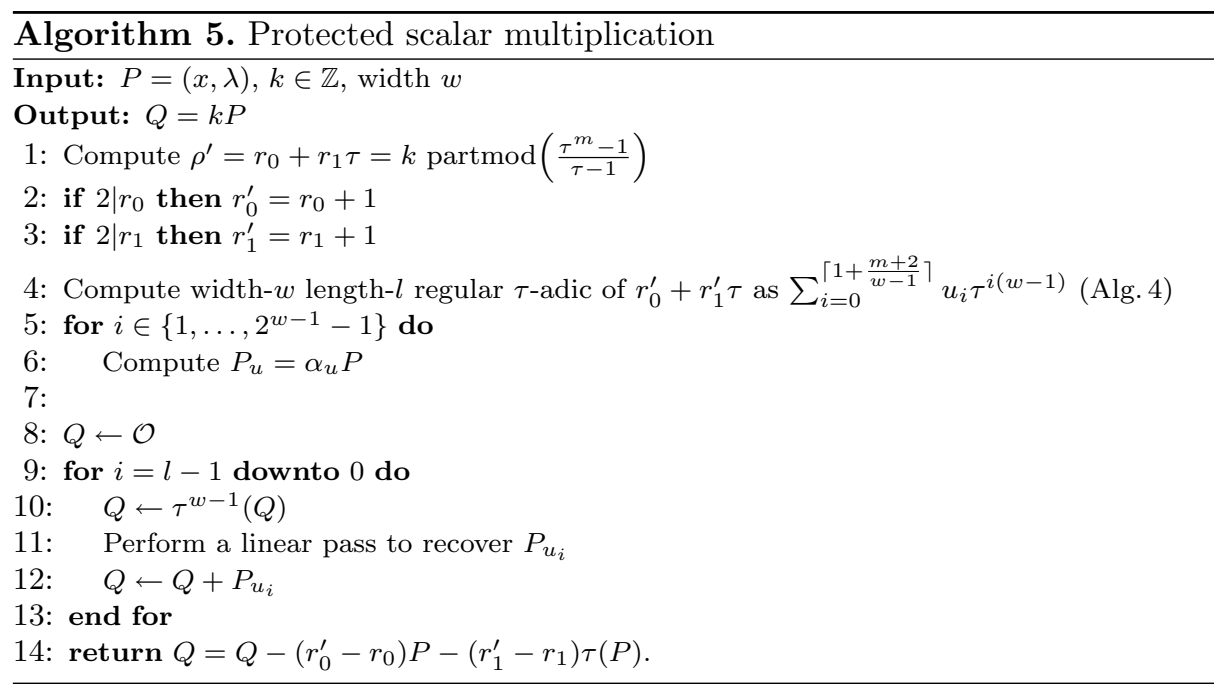




\section{Implementation Issues and Results}

In this Section, we discuss several implementation issues. We also present our experimental results and we compare them against state-of-the-art protected point multiplication implementations at the 128-bit security level.

\subsection{Mechanisms to Achieve a Constant-Time GLS-Montgomery Ladder Implementation}

To protect the previously described algorithms against timing attacks, we observed the following precautions:

Branchless Code. The main loop, the pre- and post-computation phases are implemented by a completely branch-free code.

Data Veiling. To guarantee a constant memory access pattern in the main loop of the Montgomery ladder algorithms, we proposed an efficient data veiling method, as described in Algorithm 7 of Appendix B. Algorithm 7 evaluates the actual and the previous scalar bits to decide whether the variables containing the Montgomery-LD accumulators values should or should not be masked. This strategy saves a considerable portion of the computational effort associated to Algorithm 1 of [4].

Field Arithmetic. Two of the base field arithmetic operations over $\mathbb{F}_{q}$ were implemented through look-up tables, namely, the half-trace and the multiplicative inverse operations. The half-trace is used to perform the point halving primitive, which is required in the pre-computation phase of the Montgomery-LD halve-and-add algorithm. The multiplicative inverse is one of the operations in the $y$-coordinate retrieval procedure, at the end of the Montgomery ladder algorithms. Also, whenever post-computational additions are necessary, inverses must be performed to convert a point from LD-projective to affine coordinates.

Although we are aware of the existence of protocols that consider the base point as a secret information [6], in which case one could not consider that our software provides protection against timing attacks, in the vast majority of protocols, the base point is public. Consequently, any attacks aimed at the two field operations mentioned above would be pointless.

\subsection{Mechanisms to Achieve a Constant-Time Koblitz Implementation}

Implementing Algorithm 5 in constant time needs some care, since all of its building blocks must be implemented in constant time.

Finite Field Arithmetic. Modern implementations of finite field arithmetic can make extensive use of vector registers, removing timing variances due to the cache hierarchy. For our illustrative implementation of curve NIST-K283, we closely follow the arithmetic described in Bluhm-Gueron [4], adopting the incomplete reduction improvement proposed by Negre-Robert [28]. 
Integer Recoding. All the branches in Algorithm 4 need to be eliminated by conditional execution statements to protect leakage of the scalar $k$. Moreover, to remove the remaining sign-related branches, multiple precision integer arithmetic must be implemented in complement of two. If two constants, say $\beta_{u}, \gamma_{u}$, are stored in a precomputed table, then they need to be recovered by a linear pass across the table in constant time. Finally, the partial reduction step producing $\rho^{\prime}$ must also be implemented in constant time by removing all of its branches. Notice that the requirement for $r_{0}, r_{1}$ to be odd is not a problem, since partial reduction can be modified to always result in odd integers, with a possible correction at the end of the scalar multiplication by performing a (protected) conditional subtraction of points (line 14 of Algorithm 5).

\subsection{Results}

Our implementation was mainly designed for the Intel Haswell processor family, which supports vectorial sets such as SSE and AVX, a carry-less multiplication and some bit manipulation instructions. The programming was done in $\mathrm{C}$ with the support of assembly inline code. The compilation was performed via GCC version 4.7.3 with the flags $-\mathrm{m} 64-$ march=core-avx2 -mtune=core-avx2 -03 -fomit-frame-pointer -funroll-loops. Finally, the timings were collected on an Intel Core i7-4700MQ, with the Turbo Boost and Hyperthreading features disabled $^{4}$.

Table 2 presents the experimental timings obtained for the most prominent building blocks required for computing the point multiplication operation on the GLS and Koblitz binary elliptic curves.

We present in Table 3 a comparison of our timings against a selection of state-of-the-art implementations of the point multiplication operation on binary and prime elliptic curves. Due to the Montgomery-LD point doubling efficiency, which costs $49 \%$ less than a point halving, the GLS-Montgomery-LD-doubleand-add achieved the fastest timing in the one-core setting, with 70,800 clock cycles. This is $13 \%$ faster than the performance obtained by the GLS-Montgomery-LD-halve-and-add algorithm. In the known-base point setting, we can ignore the GLS-Montgomery-LD-halve-and-add pre-computation expenses associated with its table of halved points. In that case, we can compute the scalar multiplication in an estimated time of 44,600 clock cycles using a table of just 4128 bytes.

${ }^{4}$ We intend to submit our software to the ECRYPT Benchmarking of Cryptographic Systems (eBACS) SUPERCOP toolkit in the near future. 
Table 2. Timings (in clock cycles) for the elliptic curve operations in the Intel Haswell platform.

\begin{tabular}{|c|c|c|}
\hline \multirow{2}{*}{$\begin{array}{l}\text { Elliptic curve } \\
\text { operation }\end{array}$} & \multicolumn{2}{|c|}{ GLS $E / \mathbb{F}_{2^{254}}$} \\
\hline & cycles & $o p / M^{1}$ \\
\hline Halving & 184 & 4.181 \\
\hline Montgomery-LD D\&A (left-to-right) Addition (Eq. (6)) & 161 & 3.659 \\
\hline Montgomery-LD H\&A (right-to-left) Addition (Eq. (8)) & 199 & 4.522 \\
\hline Montgomery-LD Doubling ${ }^{\mathrm{a}}$ (Eq. (5)) & 95 & 2.159 \\
\hline
\end{tabular}

\begin{tabular}{|c|c|c|c|}
\hline \multirow{2}{*}{\begin{tabular}{|l|} 
Elliptic curve \\
operation
\end{tabular}} & & \multicolumn{2}{|c|}{ Koblitz $E / \mathbb{F}_{2^{283}}$} \\
\hline & & cycles & $o p / M^{1}$ \\
\hline & Frobenius & 70 & 1.235 \\
\hline & Integer $\tau$-adic recoding (Alg. 4$)(w=5)$ & 8,900 & 156.863 \\
\hline & Point addition & 602 & 10.588 \\
\hline
\end{tabular}

${ }^{1}$ Ratio to multiplication.

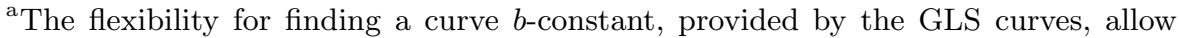
us to have a small $\sqrt{b}$ (see Appendix C). As a consequence, we used the Eq. (5) alternative formula.

Furthermore, the GLS-Montgomery-LD-halve-and-add is crucial for implementing the multi-core versions of the Montgomery ladder. When compared with our one-core double-and-add implementation, Table 3 reports a speedup of 1.36 and 2.03 in our two- and four-core Montgomery ladder versions, respectively. Here, besides the overhead costs commented in Sect. 3, we can clearly perceive the usual multicore management penalty. Finally, we observe that our GLSMontgomery-LD-double-and-add surpasses by $48 \%, 40 \%$ and $2 \%$ the Montgomery ladder implementations of [4] (Random), [4] (Koblitz) and [1], respectively.

As for our Koblitz implementations, the fast $\tau$ endomorphism allows us to have a regular-recoding implementation that outperforms a standard Montgomery ladder for Koblitz curves by $18 \%$. In addition, our fastest Koblitz code surpasses by $16 \%$ the recent implementation reported in $[4]^{5}$. Finally, note that, in spite of the fact that the $\tau$ endomorphism is $26 \%$ faster than the MontgomeryLD point doubling, the superior efficiency of the GLS quadratic field arithmetic produces faster results for the GLS Montgomery ladder algorithms.

\footnotetext{
${ }^{5}$ We could not reproduce the timing of 118,000 cycles with the code available from [4], which indicates that TurboBoost could be possibly turned on their benchmarks. Considering this, our implementation of Koblitz-Montgomery-LD becomes $9 \%$ faster than [4], reflecting the savings from partial reduction, and the speedup achieved by the Koblitz-regular implementation increases to $26 \%$.
} 
Table 3. Timings (in clock cycles) for 128-bit level scalar multiplication with timingattack resistance in the Intel Ivy Bridge (I) and Haswell (H) architectures.

\begin{tabular}{|c|c|c|c|}
\hline & Method & Cycles & Arch \\
\hline \multirow{7}{*}{ 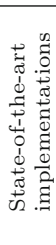 } & Montgomery-DJB-chain (prime) [7] & 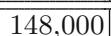 & 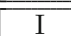 \\
\hline & Random-Montgomery-LD ladder (binary) [4] & 135,000 & $\mathrm{H}$ \\
\hline & Genus-2-Kummer (prime) [5] & 122,000 & I \\
\hline & Koblitz-Montgomery-LD ladder (binary) [4] & 118,000 & $\mathrm{H}$ \\
\hline & Twisted-Edwards-4-GLV (prime) [11] & 92,000 & I \\
\hline & Genus-2-Kummer Montgomery ladder (prime) [1] & 72,200 & $\mathrm{H}$ \\
\hline & GLS-2-GLV double-and-add (binary, $\lambda$ ) [32] & 60,000 & $\mathrm{H}$ \\
\hline \multirow{6}{*}{ 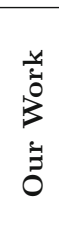 } & Koblitz-Montgomery-LD double-and-add (left-to-right) & 122,000 & $\mathrm{H}$ \\
\hline & Koblitz-regular $\tau$-and-add (left-to-right, $w=5$ ) & 99,000 & $\mathrm{H}$ \\
\hline & GLS-Montgomery-LD-2-GLV halve-and-add (Algorithm 3) & 80,800 & $\mathrm{H}$ \\
\hline & GLS-Montgomery-LD double-and-add (Algorithm 1) & 70,800 & $\mathrm{H}$ \\
\hline & 2-core GLS-Montgomery-LD-2-GLV halve-and-add/double-and-add & 52,000 & $\mathrm{H}$ \\
\hline & $\begin{array}{l}\text { 4-core GLS-Montgomery-LD-2-GLV halve-and-add/double-and-add } \\
\text { (Algorithm 6) }\end{array}$ & 34,800 & $\mathrm{H}$ \\
\hline
\end{tabular}

\section{Conclusion}

We presented several algorithms that permit to compute a constant-time highsecurity point multiplication operation over two families of binary elliptic curves, namely, the GLS and the Koblitz curves. Although this work was completely focused on a high-end desk computation of the variable-base point multiplication, the possibility of applying Algorithm 3 to the fixed-base point multiplication setting is highly appealing since that procedure requires a comparatively small pre-computed table of roughly $2 n \cdot(n+1)$ bits for computing a point multiplication at the $n$-bit security level. The above combined with the Montgomery ladder unique feature of performing all the computations using only two point coordinates, should be attractive for deployments of public key cryptography on constrained computing environments.

\section{A Multi-core Montgomery Ladder}

Here we present the four-core GLS-Montgomery-LD ladder algorithm. Given $t_{4}$ the integer constant that establishes the workload of each algorithm, $P \in E\left(\mathbb{F}_{q^{2}}\right)$, and the scalar $k$ represented as $k_{1}+k_{2} \cdot \delta$ using the GLS-GLV method, cores $I$ and $I I$ are both responsible for computing $\left\lfloor\frac{n}{2}\right\rfloor-t_{4}$ bits of the subscalars $k_{1}$ and $k_{2}$ using the Montgomery-LD double-and-add method. In turn, the cores $I I I$ and $I V$, both compute $t_{4}$ bits of $k_{1}$ and $k_{2}$ with the Montgomery-LD halveand-add algorithm. In the end, on a single core, it is necessary to add all the accumulators $Q_{i}$, for $i=0 \ldots 3$. 


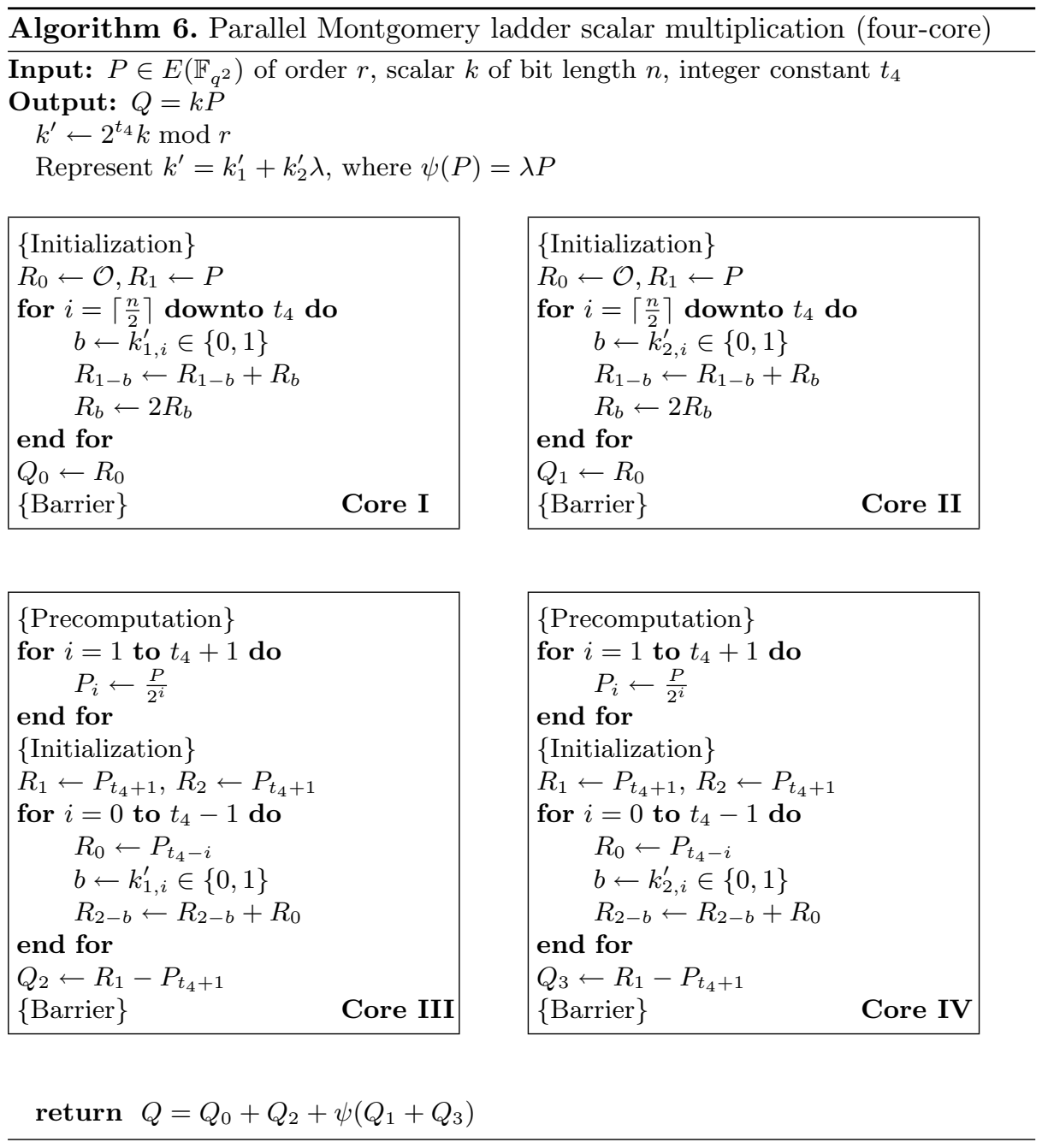

\section{B Memory Access Pattern}

The following data veiling algorithm ensures a fixed memory access pattern for all Montgomery-LD ladder algorithms. Given the two Montgomery-LD ladder accumulators $A$ and $B$, and the scalar $k=\left(k_{n-1}, k_{n-2}, \ldots k_{0}\right)$, this method allows us, in the beginning of the $i$-th main loop iteration, to use the bits $k_{i-1}$ and $k_{i}$ to decide if $A$ and $B$ will or will not be swapped. As a result, it is not necessary to reapply the procedure at the end of the $i$-th iteration. 


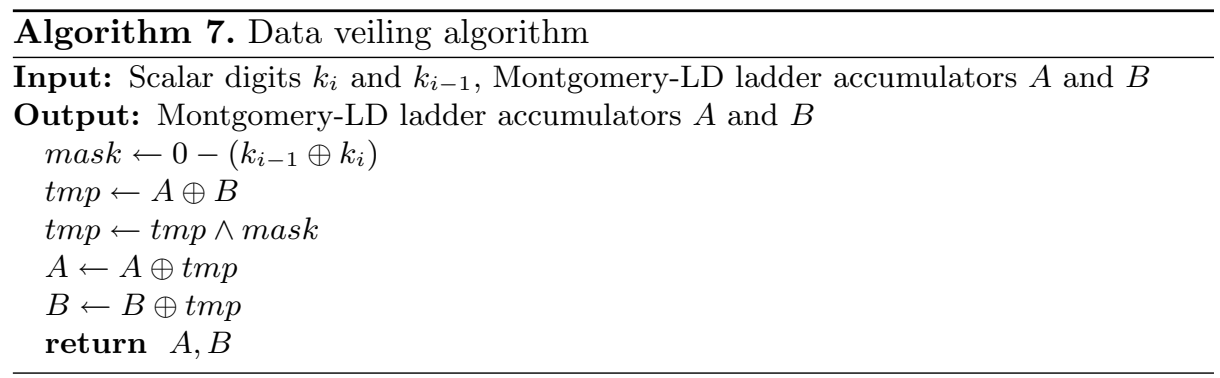

\section{GLS Elliptic Curve Parameters}

For achieving a greater benefit from the multiplication by the $b$-constant in the Montgomery-LD doubling formula $X_{3}=X_{0}{ }^{4}+b Z_{0}{ }^{4}=\left(X_{0}{ }^{2}+\sqrt{b} Z_{0}{ }^{2}\right)^{2}$ we carefully selected a GLS curve with a 64-bit $b$-parameter square-root. As a result, we saved two carry-less multiplication and a dozen of SSE instructions per field multiplication. Next, we describe the parameters, as polynomials represented in hexadecimal, for our GLS curve $E_{a, b} / \mathbb{F}_{q^{2}}: y^{2}+x y=x^{3}+a x^{2}+b$.

$-a=u$

$-b=0 \times 54045144410401544101540540515101$

$-\sqrt{b}=0 x E 2 D A 921 E 91 E 38 D D 1$

The 253-bit prime order $r$ of the main subgroup of $E_{a, b} / \mathbb{F}_{q^{2}}$ is,

$r=$ 0x1FFFFFFFFFFFFFFFFFFFFFFFFFFFFFFA6B89E49D3FECD828CA8D66BF4B88ED5.

Also, the integer $\delta$ such that $\psi(P)=\delta P$ for all $P \in E_{a, b}$ is,

$\delta=$ 0x74AEFB81EE8A42E9E9D0085E156A8EFBA3D302F9C74D737FA00360F9395C788.

The base point $P=(x, y)$ of order $r$ used in this work is,

$x=0 \times 4$ A21A3666CF9CAEBD812FA19DF9A3380 + 0x358D7917D6E9B5A7550B1B083BC299F3 $\cdot u$

$y=0 \times 6690$ CB7B914B7C4018E7475D9C2B1C13 + 0x2AD4E15A695FD54011BA179D5F4B44FC $\cdot u$.

Finally, the towering of our field $\mathbb{F}_{q} \cong \mathbb{F}_{2}[x] /(f(x))$ and its quadratic extension $\mathbb{F}_{q^{2}} \cong \mathbb{F}_{q}[u] /(g(x))$ is constructed by means of the irreducible trinomials $f(x)=$ $x^{127}+x^{63}+1$ and $g(u)=u^{2}+u+1$.

\section{References}

1. Bernstein, D.J., Chuengsatiansup, C., Lange, T., Schwabe, P.: Kummer strikes back: new DH speed records. Cryptology ePrint Archive, Report 2014/134 (2014). http://eprint.iacr.org/

2. Bernstein, D.J., Lange, T.: SafeCurves: choosing safe curves for elliptic-curve cryptography. http://safecurves.cr.yp.to 
3. Bernstein, D.J., Lange, T.: Security dangers of the NIST curves. Invited talk, International State of the Art Cryptography Workshop, Athens, Greece (2013)

4. Bluhm, M., Gueron, S.: Fast software implementation of binary elliptic curve cryptography. Cryptology ePrint Archive, Report 2013/741 (2013). http://eprint. iacr.org/

5. Bos, J.W., Costello, C., Hisil, H., Lauter, K.: Fast cryptography in genus 2. In: Johansson, T., Nguyen, P.Q. (eds.) EUROCRYPT 2013. LNCS, vol. 7881, pp. 194-210. Springer, Heidelberg (2013)

6. Chatterjee, S., Karabina, K., Menezes, A.: A new protocol for the nearby friend problem. In: Parker, M.G. (ed.) Cryptography and Coding 2009. LNCS, vol. 5921, pp. 236-251. Springer, Heidelberg (2009)

7. Costello, C., Hisil, H., Smith, B.: Faster compact Diffie-Hellman: endomorphisms on the $x$-line. In: Nguyen, P.Q., Oswald, E. (eds.) EUROCRYPT 2014. LNCS, vol. 8441, pp. 183-200. Springer, Heidelberg (2014)

8. Dierks, T., Allen, C.: The TLS Protocol Version 1.0. RFC 2246 (Proposed Standard), January 1999. Obsoleted by RFC 4346, updated by RFCs 3546, 5746, 6176

9. Dierks, T., Rescorla, E.: The Transport Layer Security (TLS) Protocol Version 1.1. RFC 4346 (Proposed Standard), April 2006. Obsoleted by RFC 5246, updated by RFCs 4366, 4680, 4681, 5746, 6176

10. Dierks, T., Rescorla, E.: The Transport Layer Security (TLS) Protocol Version 1.2. RFC 5246 (Proposed Standard), August 2008

11. Faz-Hernández, A., Longa, P., Sánchez, A.H.: Efficient and secure algorithms for GLV-based scalar multiplication and their implementation on GLV-GLS curves. In: Benaloh, J. (ed.) CT-RSA 2014. LNCS, vol. 8366, pp. 1-27. Springer, Heidelberg (2014)

12. Fog, A.: Instruction Tables: List of Instruction Latencies, Throughputs and Microoperation Breakdowns for Intel, AMD and VIA CPUs. http://www.agner.org/ optimize/instruction_tables.pdf. Accessed 14 May 2014

13. Galbraith, S.D., Lin, X., Scott, M.: Endomorphisms for faster elliptic curve cryptography on a large class of curves. In: Joux, A. (ed.) EUROCRYPT 2009. LNCS, vol. 5479, pp. 518-535. Springer, Heidelberg (2009)

14. Gallant, R.P., Lambert, R.J., Vanstone, S.A.: Faster point multiplication on elliptic curves with efficient endomorphisms. In: Kilian, J. (ed.) CRYPTO 2001. LNCS, vol. 2139, pp. 190-200. Springer, Heidelberg (2001)

15. Gaudry, P., Hess, F., Smart, N.P.: Constructive and destructive facets of Weil descent on elliptic curves. J. Cryptol. 15, 19-46 (2002)

16. Gueron, S., Krasnov, V.: Fast prime field elliptic curve cryptography with 256 bit primes. Cryptology ePrint Archive, Report 2013/816 (2013). http://eprint. iacr.org/

17. Hankerson, D., Karabina, K., Menezes, A.: Analyzing the Galbraith-Lin-Scott point multiplication method for elliptic curves over binary fields. IEEE Trans. Comput. 58(10), 1411-1420 (2009)

18. Hankerson, D., Menezes, A., Vanstone, S.: Guide to Elliptic Curve Cryptography. Springer, Secaucus (2003)

19. Hess, F.: Generalising the GHS attack on the elliptic curve discrete logarithm problem. LMS J. Comput. Math. 7, 167-192 (2004)

20. Huang, Y.-J., Petit, C., Shinohara, N., Takagi, T.: Improvement of Faugère et al.'s method to solve ECDLP. In: Sakiyama, K., Terada, M. (eds.) IWSEC 2013. LNCS, vol. 8231, pp. 115-132. Springer, Heidelberg (2013) 
21. Joye, M., Tunstall, M.: Exponent recoding and regular exponentiation algorithms. In: Preneel, B. (ed.) AFRICACRYPT 2009. LNCS, vol. 5580, pp. 334-349. Springer, Heidelberg (2009)

22. Koblitz, A.H., Koblitz, N., Menezes, A.: Elliptic curve cryptography: the serpentine course of a paradigm shift. J. Number Theory 131(5), 781-814 (2011)

23. Koblitz, N.: CM-curves with good cryptographic properties. In: Feigenbaum, J. (ed.) CRYPTO 1991. LNCS, vol. 576, pp. 279-287. Springer, Heidelberg (1992)

24. Longa, P., Sica, F.: Four-dimensional Gallant-Lambert-Vanstone scalar multiplication. J. Cryptol. 27(2), 248-283 (2014)

25. López, J., Dahab, R.: Fast multiplication on elliptic curves over $\mathrm{GF}\left(2^{\mathrm{m}}\right)$ without precomputation. In: Koç, Ç.K., Paar, C. (eds.) CHES 1999. LNCS, vol. 1717, pp. 316-327. Springer, Heidelberg (1999)

26. Montgomery, P.L.: Speeding the Pollard and elliptic curve methods of factorization. Math. Comput. 48, 243-264 (1987)

27. National Institute of Standards and Technology. Recommended Elliptic Curves for Federal Government Use. NIST Special Publication (1999). http://csrc.nist.gov/ csrc/fedstandards.html

28. Négre, C., Robert, J.-M.: Impact of optimized field operations $A B, A C$ and $A B+C D$ in scalar multiplication over binary elliptic curve. In: Youssef, A., Nitaj, A., Hassanien, A.E. (eds.) AFRICACRYPT 2013. LNCS, vol. 7918, pp. 279-296. Springer, Heidelberg (2013)

29. Nègre, C., Robert, J.-M.:. New parallel approaches for scalar multiplication in elliptic curve over fields of small characteristic (2013). http://hal.archives-ouvertes. $\mathrm{fr} /$ docs $/ 00 / 90 / 84 / 63 / \mathrm{PDF} /$ parallelization-ecsm8.pdf

30. Okeya, K., Takagi, T., Vuillaume, C.: Efficient representations on Koblitz curves with resistance to side channel attacks. In: Boyd, C., González Nieto, J.M. (eds.) ACISP 2005. LNCS, vol. 3574, pp. 218-229. Springer, Heidelberg (2005)

31. Oliveira, T., López, J., Aranha, D.F., Rodríguez-Henríquez, F.: Lambda coordinates for binary elliptic curves. In: Bertoni, G., Coron, J.-S. (eds.) CHES 2013. LNCS, vol. 8086, pp. 311-330. Springer, Heidelberg (2013)

32. Oliveira, T., López, J., Aranha, D.F., Rodríguez-Henríquez, F.: Two is the fastest prime: lambda coordinates for binary elliptic curves. J. Cryptogr. Eng. 4(1), 3-17 (2014)

33. Petit, C., Quisquater, J.-J.: On polynomial systems arising from a Weil descent. In: Wang, X., Sako, K. (eds.) ASIACRYPT 2012. LNCS, vol. 7658, pp. 451-466. Springer, Heidelberg (2012)

34. Salowey, J.: Confirming consensus on removing RSA key transport from TLS 1.3. Transport Layer Security Working Group of the IETF Mailing List, 3 May 2014

35. Scott, M.: Re: NIST announces set of elliptic curves (1999). https://groups.google. com/forum/message/raw?msg=sci.crypt/mFMukSsORmI/FpbHDQ6hM_MJ

36. Shantz, M., Teske, E.: Solving the elliptic curve discrete logarithm problem using Semaev polynomials, Weil descent and Gröbner basis methods - an experimental study. Cryptology ePrint Archive, Report 2013/596 (2013). http://eprint.iacr.org/

37. Solinas, J.A.: Efficient arithmetic on Koblitz curves. Des. Codes Crypt. 19(2-3), 195-249 (2000)

38. Taverne, J., Faz-Hernández, A., Aranha, D.F., Rodríguez-Henríquez, F., Hankerson, D., López, J.: Speeding scalar multiplication over binary elliptic curves using the new carry-less multiplication instruction. J. Cryptogr. Eng. 1, 187-199 (2011) 